\title{
Canine transmissible venereal tumor: First report of three clinical cases from Tripoli, Libya
}

\author{
Murad A. Hiblu' ${ }^{1}$ Nizar M. Khabuli ${ }^{2,3}$ and Abdurraouf O. Gaja ${ }^{2, *}$ \\ ${ }^{1}$ Department of Internal Medicine, Faculty of Veterinary Medicine, University of Tripoli, Tripoli, Libya \\ ${ }^{2}$ Department of Veterinary Surgery and Theriogenology, Faculty of Veterinary Medicine, University of Tripoli, \\ Tripoli, Libya \\ ${ }^{3}$ Police K9 Department, CID, Libyan Ministry of Interior, Tripoli, Libya
}

\begin{abstract}
Canine transmissible venereal tumour (CTVT) is frequently reported in dogs and is responsible for high morbidity rates and economic losses. Three clinical cases were presented at the clinic of the Faculty of Veterinary Medicine, University of Tripoli. One male and two female German shepherds were diagnosed with CTVT based on case history and tumor shape. The diagnosis was confirmed by histopathological examination. The dogs were treated with vincristine intravenously at a dose of $0.025 \mathrm{mg} / \mathrm{kg}$ and recovered fully within 4 weeks. All three dogs remained alive with no evidence of recurrence. These first cases of CTVT reported from Libya show the importance of combining case history, clinical examination and laboratory confirmation to arrive at a definitive diagnosis and implement effective therapy. Keywords: Dogs, Oncology, Transmissible venereal tumor, Vincristine.
\end{abstract}

\section{Introduction}

Canine transmissible venereal tumor (CTVT) is a contagious venereal tumor with round-cell tumor of mesenchymal origin. The disease primarily observed on mucosal surface of the external genitalia of male and female dogs. Venereal transmission is the most common way of infection, but it can be transplanted to other sites and transmitted to other dogs by licking, biting, and by direct contact with the tumor (Kabuusu et al., 2010; Ostrander et al., 2016).

\section{Case Details}

Two females and one male German shepherd were presented separately to Albaittar veterinary clinic, Tripoli, Libya and were transferred to the Faculty of Veterinary Medicine, Tripoli, Libya. The ages of two females were 2 and 4-yr old, respectively, and the male was 2.5-yr old. The history revealed a slight decrease in appetite, weight loss, infertility, and progressive cauliflower like tumorous vaginal growth in both females, and fresh drops of blood oozing from the prepuce of the male. Both of the females were mated by the same affected male. One of the female was surgically treated and resubmitted for the recurrence. Duration of illness was almost 2 and 3 mo, respectively, in females and 1 mo in male. The clinical examination revealed lethargy, anemia, weight loss, and fleshy hypermic, cauliflower like growth on the vulva and in the vagina of both females (Fig. 1A and $\mathrm{B}$ ), and similar growth was found on the bulbus glandis area of the penis of the affected male (Fig. 2).
The growths were quite friable and bleed easily on manipulation. The diagnosis was strongly based on the clinical examination and confirmed by examination of hematoxylin- and eosin-stained sections of the masses. Microscopic examination revealed sheets of malignant cells infiltration. The malignant cells were large in size with large hyperchromatic pleomorphic nuclei and prominent nuclei with frequent mitosis, surrounded by dermoplastic stroma. The presence of identical large, vacuolated tumor cells (Fig. 3) confirmed the diagnosis. The three cases were treated with intravenous injections of Vincristine at the rate dose of $0.025 \mathrm{mg} / \mathrm{kg}$ once a week for $1 \mathrm{mo}$. The drug was diluted in $250 \mathrm{ml}$ of
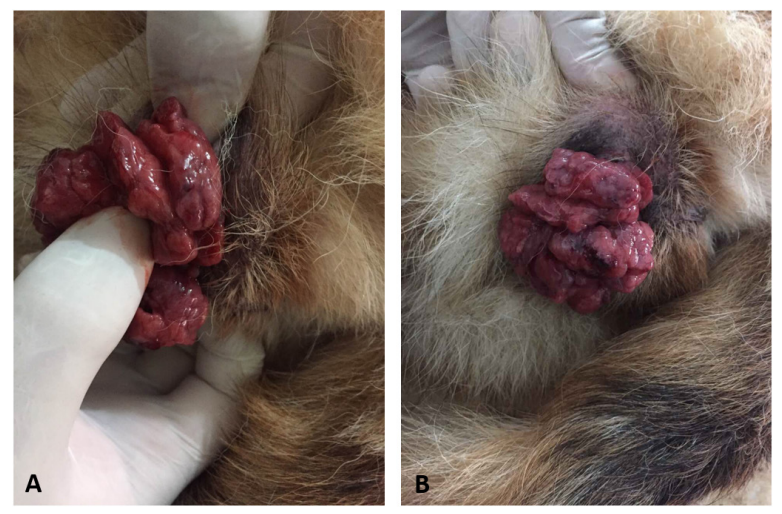

Fig. 1. (A) Female external genitalia infected with CTVT (Case 1). (B) Female external genitalia infected with CTVT (Case 2). 
normal saline and administered slowly over half an hour.

On treatment with Vincristine, all three dogs showed complete regression of the tumor mass. No tumor recurrence was noted. The female dogs showed complete regression after 4 wk of treatment, while the male dog

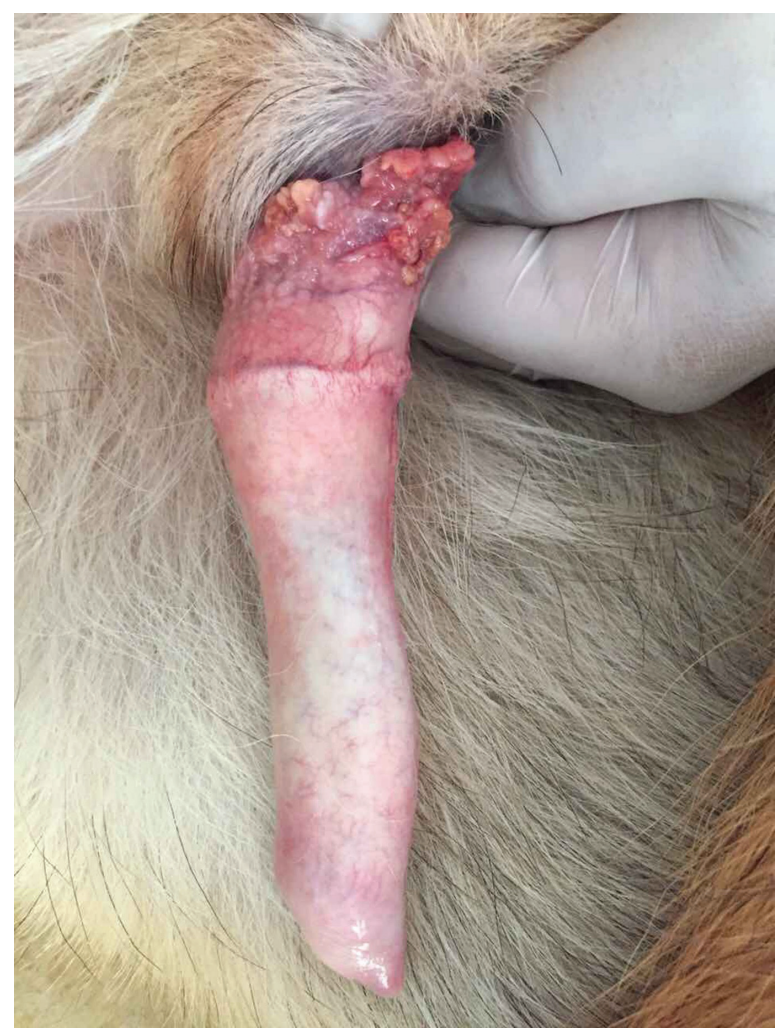

Fig. 2. Male external genitalia infected with CTVT (Case 3).

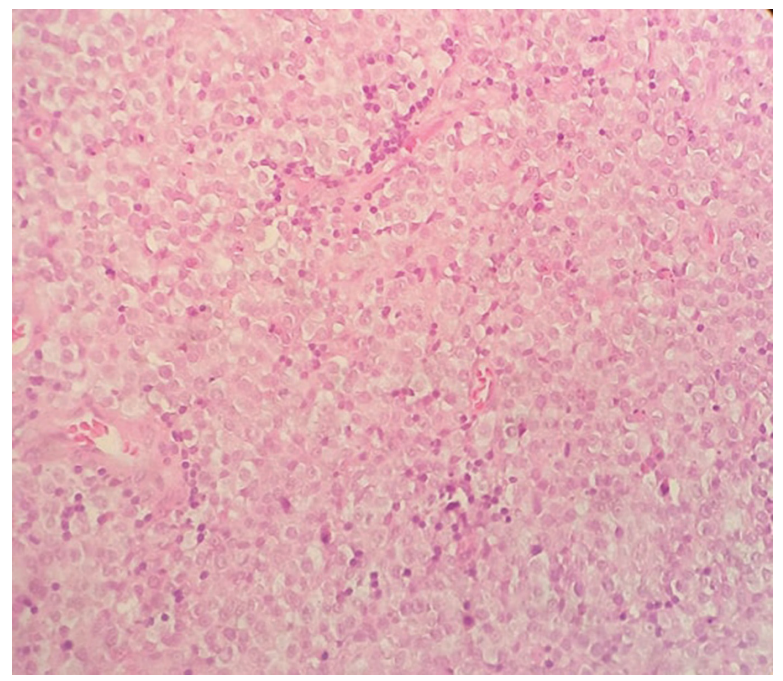

Fig. 3. Hematoxylin- and eosin-stained section of an untreated canine transmissible venereal tumor showing the large vacuolated tumor cells $(\times 40)$. was cured after three injections. Interestingly, all three dogs remained in good health condition with no evidence of tumor recurrence was noted and one of the treated females was mated, became pregnant, and whelped four pups, and her pups were in good health condition.

\section{Discussion}

CTVTs feature strong genetic identity with one another but are markedly distinct from their extant, transient host (Murchison et al., 2014; Decker et al., 2015). CTVT is endemic in more than 90 countries and believed to be the most widely disseminated tumor in existence (Das and Das, 2000; Murchison, 2008; Strakova and Murchison, 2014; Ganguly et al., 2016). Recent data suggest that CTVT has likely existed on all continents (except Antarctica) for decades (Ganguly et al., 2016). To our knowledge, the present finding is the first clinical report of CTVT in dogs reported in Libya. No data have been reported on the incidence of CTVT in local breed dogs, and all the affected cases were from the offspring's of German shepherd, which were imported from abroad several years ago. Our study confirms that the treatment regimen of intravenous administration of vincristine sulfate at a dose of $0.025 \mathrm{mg} / \mathrm{kg}$ body weight once a week for $4 \mathrm{wk}$ was very effective in the complete remission of CTVT in dogs (male and female), and these results are consistent with previously published data (Tella et al., 2004; Antonov, 2015). In our study, only anorexia as a side effect of vincristine sulfate was observed in one dog for 4 days at the commence of treatment, which is a common phenomenon of cancer chemotherapy. Cross breeding of different breed dogs has become a common phenomenon in Libya nowadays among the dog breeders; therefore, there is a greater risk that the disease will become prevalent in Libya. A high level of veterinary surveillance is required to control the disease.

\section{Acknowledgments}

The authors are grateful to Dr. Khawla Ejnan for her valuable laboratory work, and to Dr. Mohamed O. Ahmed for his scientific contribution. Also, they are thankful for the staff of Albaittar veterinary clinic for their assistance and cooperation.

\section{Conflict of interest}

The authors declare that there is no conflict of interest.

\section{References}

Antonov, A. 2015. Successful treatment of canine transmissible venereal tumor using vincristine sulfate. Adv. Res. 5, 1-5.

Das, U. and Das, A.K. 2000. Review of canine transmissible venereal sarcoma. Vet. Res. Commun. 24, 545-556.

Decker, B., Davis, B.W., Rimbault, M., Long, A.H., Karlins, E., Jagannathan, V., Reiman, R., Parker, 
H.G., Drögemüller, C., Corneveaux, J.J., Chapman, E.S., Trent, J.M., Leeb, T., Huentelman, M.J., Wayne, R.K., Karyadi, D.M. and Ostrander, E.A. 2015. Comparison against 186 canid whole-genome sequences reveals survival strategies of an ancient clonally transmissible canine tumor. Genome Res. 25, 1646-1655.

Ganguly, B., Das, U. and Das, A.K. 2016. Canine transmissible venereal tumour: a review. Vet. Comp. Oncol. 14, 1-12.

Kabuusu, R.M., Stroup, D.F. and Fernandez, C. 2010. Risk factors and characteristics of canine transmissible venereal tumours in Grenada, West Indies. Vet. Comp. Oncol. 8, 50-55.

Murchison, E.P. 2008. Clonally transmissible cancers in dogs and Tasmanian devils. Oncogene 27(Suppl. 2), S19-S30.

Murchison, E.P., Wedge, D.C., Alexandrov, L.B., Fu, B., Martincorena, I., Ning, Z., Tubio, J.M.C.,
Werner, E.I., Allen, J., De Nardi, A.B., Donelan, E.M., Marino, G., Fassati, A., Campbell, P.J., Yang, F., Burt, A., Weiss, R.A. and Stratton, M.R. 2014. Transmissible [corrected] dog cancer genome reveals the origin and history of an ancient cell lineage. Science 343, 437-440.

Ostrander, E.A., Davis, B.W. and Ostrander, G.K. 2016. Transmissible tumors: breaking the cancer paradigm. Trends Genet. 32, 1-15.

Strakova, A. and Murchison, E.P. 2014. The changing global distribution and prevalence of canine transmissible venereal tumour. BMC Vet. Res. $10,168$.

Tella, M.A., Ajala, O.O. and Taiwo, V.O. 2004. Complete regression of transmissible venereal tumor (TVT) in Nigerian mongrel dogs with vincristine sulphate chemotherapy. Afr. J. Biomed. Res. 7, 133-138. 